\title{
Assessing the Work Practices and Information Needs of Disease Investigators
}

\author{
Neil Abernethy*, Lauren Carroll, Alan Au and Tsung-Chieh Fu \\ Biomedical Informatics and Medical Education, University of Washington, Seattle, WA, USA
}

\section{Objective}

The goal of this work is to identify specific work practices in disease investigation that would be supported by data visualization, such as identifying exposure, contact, and spatiotemporal clustering.

\section{Introduction}

Investigation of cases, clusters, and outbreaks of infectious disease is a complex process requiring substantial support from protocols, distributed and cooperative work, and information systems. We set out to identify public health information needs, the types of data required to meet these needs, and the potential alignment with visualizations of this data.

\section{Methods}

We used mixed methods to identify common high-level themes and low-level data elements required during disease investigation. We first studied disease investigation protocols and contact investigation data forms from 31 jurisdictions in the context of tuberculosis case investigations.

To provide further insight into the disease investigation process, we conducted qualitative research with a public health department to identify work practices, protocols, paper forms, electronic tools, visualizations, and information needs encountered during case and outbreak investigations. This research consisted of two focus groups with different divisions of a public health department, attendance of daily meetings, and participant observation of investigators, nurses, and epidemiologists in the Communicable Disease Section.

\section{Results}

We obtained 25 data forms and 21 protocols from 31 states representing contact investigation practices. Both forms and protocols were available from 15 states, allowing comparison of the data fields in these artifacts. Of 82 data elements recorded, only 10 were commonly represented in both data forms and protocols. This suggests that not all work practices were encoded in these protocols, that some protocoldriven activities are not reflected in the forms we analyzed, and that the functions of these artifacts are not well coordinated.

Disease investigation practices vary widely by jurisdiction, however as expected some data fields (e.g. demographics, transmission risk, and clinical/case management variables) are more common across jurisdictions. Similarly, some common functionality is required of information systems, such as patient identification and search, diagnosis, treatment monitoring, tracking trends, and identifying or plotting links between cases and those at risk.

We used qualitative methods to obtain a more complete picture of disease investigation work practices that emerged from state protocols. Focus groups were used to explore several themes, including the use of information tools and how well they satisfied public health information needs. Participants routinely used information tools to record data during the disease investigation process, although existing tools were not as well-suited for data retrieval and analysis. Variations in data reporting requirements between local/state systems also affected work practices involving data entry. Participants made use of free-text data fields to record case-specific information, but this complicated extraction and analysis of these data.

Direct observation sessions reiterated the use of information systems for data management in conjunction with paper records. Consistent with findings from the data form and protocol analysis, investigators used systems to check for common risk factors that might indicate that cases were related and to track case status. Visualization might benefit such data management tasks; however most visualizations observed were not routinely available. Information systems were also used for process-related tasks, such as de-duplicating entries, accessing health records from partnering agencies, and conducting workload management tasks.

\section{Conclusions}

This research helps establish that 1) potential users of information systems have diverse workflow needs and data requirements that should be considered in system design; 2) gaps exist between the current capacity of health programs and the functions of information tools; 3) greater levels of standardization of public health data are needed within and between jurisdictions; and 4) coordinated development of data standards, infectious disease protocols, and information tools (including user interfaces and visualizations) is needed. Coordination of what public health workers should do, what they actually do, and what they could do with case data will improve future public health surveillance and response activities.

\section{Keywords}

Outbreak management; Contact investigation; Data standards; Qualitative methods; Comparison

\section{Acknowledgments}

This research was supported by NLM grant \#R01 LM011180-02. We are grateful for the participation of staff from Public Health Seattle \& King County.

\section{*Neil Abernethy \\ E-mail: neila@uw.edu}

